\title{
Channel Analysis for an OFDM-MISO Train Communications System Using Different Antennas
}

\author{
Sandra Knörzer, Michael A. Baldauf, Thomas Fügen and Werner Wiesbeck \\ Institut für Höchstfrequenztechnik und Elektronik, Universität Karlsruhe (TH) \\ Kaiserstr. 12, 76131 Karlsruhe, Germany \\ E-mail: Sandra.Knoerzer@ihe.uka.de
}

\begin{abstract}
For the design of an OFDM train communications system it is essential to characterise and consider the channel parameters. The transmission channel of a high-speed train scenario is frequency selective as well as time variant. Thus, delay spread and Doppler spread are investigated as crucial parameters for the OFDM system performance. Using a ray-tracing tool realistic impulse responses of the transmission channels are simulated. The investigated system includes three base stations operating in common frequency mode along a railway track and one mobile station situated on a high-speed train. For the mobile station different antenna patterns are included in the simulation model. The results are compared and assessed with respect to delay spread, Doppler spread and receive power. When using directional antennas a distinct reduction in Doppler spread is achieved.

Index terms - OFDM, high-speed trains, wave propagation, ray-tracing, Doppler shift, Doppler spread, directional antenna.
\end{abstract}

\section{INTRODUCTION}

A communications system based on Orthogonal Frequency Division Multiplexing (OFDM) [1] is a promising approach to provide a high data rate in a mobile environment [2]. The multi-path propagation of a transmitted signal leads to delay times that may cause intersymbol interference (ISI). This ISI can be avoided if the guard interval of an OFDM symbol is longer than the significant part of the impulse response of the transmitted signal [3], [4]. Moreover, moving mobile stations cause Doppler shift and Doppler spread within an OFDM spectrum, which can result in inter-carrier interference (ICI) [5], [6]. To diminish the ICI the subcarrier spacing of the OFDM system has to be chosen large enough. As the constraints against ISI and ICI reduce the useful data rate, a trade off has to be made between allowed ISI, ICI and the data rate.

Thus, to design a high-speed train communications system the channel properties need to be considered carefully. E.g. with a high Doppler spread of the channel the subcarrier distance of the system has to be large. If the Doppler spread can be diminished, e.g. by a directional antenna, the subcarrier distance can be reduced. This results in a higher number of subcarriers within the same bandwidth and therefore a higher data rate of the system (assuming a constant bit error rate).

In this paper a channel model for high-speed trains is used to analyse the characteristic channel parameters, especially the reduction of the Doppler spread. In Sect. II the details of the model are explained. Two typical environments are introduced as scenarios. The wave propagation is simulated using a 3D ray-tracing tool (cf. Sect. III). In Sect. IV the channel parameters for two different antennas at a mobile station and for three base stations in common-frequency mode (Multiple Input Single Output, MISO) are presented.

\section{Simulation Scenario}

The environment of high-speed trains is represented by two typical scenarios. The first scenario is called noise barrier scenario and includes a concrete floor, two pairs of metallic railway tracks, several pairs of metallic pylons and a concrete noise barrier on each side of the track. The second scenario is the vegetation scenario in which the noise barriers are replaced by trees on both sides of the track. The trees are modelled by rectangular boxes with scattering wave propagation characteristics. The distance to the railway tracks, the length, width and height of the boxes are statistically generated (Gaussian distribution).

Fig. 1 shows a schematic 2D drawing of the basic simulation environment without noise barriers or trees. The overall length of the scenario is $2000 \mathrm{~m}$. Along the railway track every $65 \mathrm{~m}$ a pair of pylons is placed facing each other. The train model complies with the German high-speed train Inter-City Express (ICE) with a length of $205 \mathrm{~m}$. On top of the train the mobile station antenna is situated. The distance from the rear part of the train 

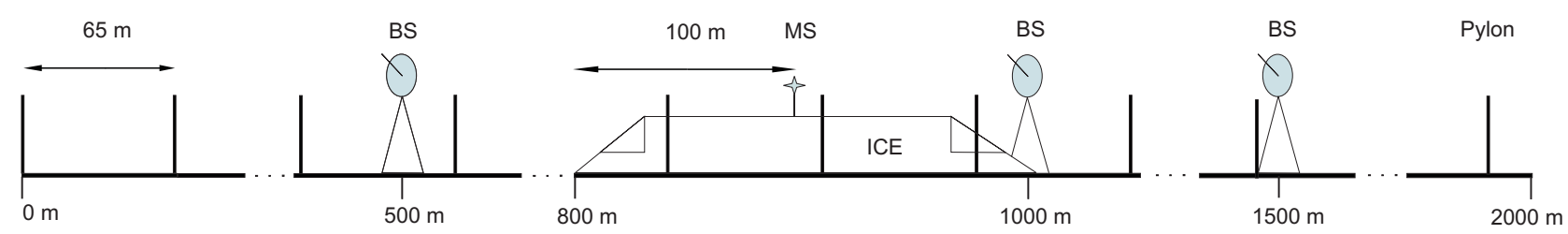

Fig. 1. Basic scenario: On top of a concrete floor, two pairs of railway tracks and every $65 \mathrm{~m}$ a pair of metallic pylons (vertical bars) are placed. Three base stations (BS) are situated at $500 \mathrm{~m}, 1000 \mathrm{~m}$ and $1500 \mathrm{~m}$. The high-speed train (ICE) moves from $800 \mathrm{~m}$ to $1000 \mathrm{~m}$ (measured at the rear part of the train). The mobile station antenna (MS) is positioned at the top of the train with a distance of $100 \mathrm{~m}$ to the rear part of the train.

to the antenna position measures $100 \mathrm{~m}$.

The presented OFDM communications system is intended to work in common-frequency mode. State-of-theart train traffic control systems are able to precisely locate the train position which can then be used to determine and switch the relevant base stations. Only the base stations next to the train send synchronised OFDM signals, e.g. the nearest three base stations. Hence, in the modelled scenario three vertically polarised omnidirectional antennas are placed as base station antennas at $500 \mathrm{~m}, 1000 \mathrm{~m}$ and $1500 \mathrm{~m}$.

In the following, first an omnidirectional and then a directional antenna are integrated as antenna for the mobile station in the channel model. Both antennas are vertically polarised. As directional antenna pattern a $\sin (\mathrm{x}) / \mathrm{x}$ characteristic is considered. This pattern type occurs as array factor in linear antenna arrays with uniform amplitude distribution [7]. The pattern data in azimuth and elevation (cf. to Fig. 2) is included in the 3D ray-tracing simulation and considered when calculating the channel parameters. In the simulation its main lobe is adjusted into the direction of travel with an antenna gain of $11.2 \mathrm{dBi}$. All channel parameters given in this paper represent the channel including the base station and mobile station antenna patterns.

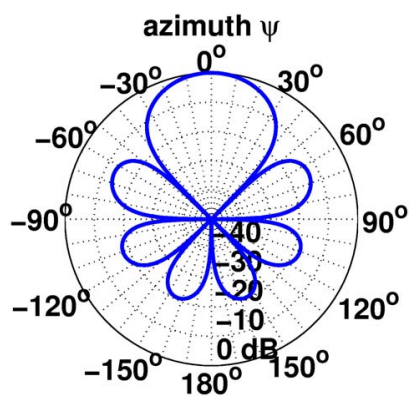

(a)

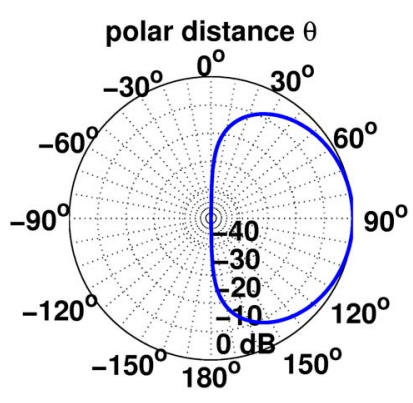

(b)
Fig. 2. Antenna pattern of the directional antenna in (a) azimuth and (b) elevation.

\section{WAVE PRopagation MOdEL}

For the wave propagation a 3D ray-tracing tool based on the theory of geometrical optics (GO) and the Uniform Theory of Diffraction (UTD) is used. The model includes modified Fresnel reflection coefficients for the reflection as well as diffraction based on the UTD. Furthermore, scattering from vegetation is added to this simulation tool. The background and implementation of this wave propagation phenomenon in the ray-tracing tool is presented in [8], [9], [10] and [11].

The major advantage of a 3D ray-tracing tool is its ability of wide-band analysis of the channel. The frequency selectivity (delay spread) and time variance (Doppler spread) of the channel can be determined. Here, especially the Doppler spread matters as this parameter influences the ICI of an OFDM system.

So far, there is no frequency allocated for a high-speed train communications system. All simulations presented in this paper are carried out in the $5 \mathrm{GHz}$ ISM band where the IEEE 802.11a wireless LAN standard is operating.

\section{Simulation Results}

In [12] simulations with directional antennas at the mobile station and one base station show that a severe reduction but also an increase of the Doppler spread compared to an omnidirectional antenna is possible. In the following, the influence of the antenna at the mobile station in a common frequency network (represented by three base stations) is investigated.

The simulation scenario is described in Section II. The train is assumed to be a perfect electric conductor (PEC) and to move uniformly with $400 \mathrm{~km} / \mathrm{h}$ along the railway tracks. The movement of the train through the scenario is simulated by a sequence of snapshots. Each snapshot represents another position of the train in the scenario and therefore another moment in time. The distance between two sequential train positions measures $1 \mathrm{~m}$ which corresponds to $9 \mathrm{~ms}$ difference in the time domain. In the first snapshot the rear part of the train is located 
at $800 \mathrm{~m}$, in the last snapshot the rear part of the train is positioned at $1000 \mathrm{~m}$ (cf. to Fig. 1).

For the scenarios considered in this paper the Doppler frequency of the incident paths at the mobile station $f_{\mathrm{D}}$ is

$$
\begin{aligned}
f_{\mathrm{D}}= & \frac{v_{\mathrm{r}}}{\lambda} \\
\text { with } & v_{\mathrm{r}}=v_{\mathrm{ICE}} \cdot \cos \varphi .
\end{aligned}
$$

Where $\lambda$ is the wavelength and $\varphi$ denotes the angle of arrival (AoA) between the direction of incidence of the propagation path and the direction of travel. $v_{\text {ICE }}$ and $v_{\mathrm{r}}$ represent the absolute velocity of the ICE and its relative velocity with respect to the direction of the incident path, respectively. Since the base station antennas and the mobile station antenna are positioned approximately at the same height, the propagation paths vary in a very small angular range in elevation. Due to this reason, the elevation component is not further discussed.

For an OFDM communications system a low Doppler spread and low delay spread is desirable. Bearing in mind the two main directions of the incident paths and the dependency of the Doppler shift on the AoA, directional antennas can e.g. attenuate paths from the backward direction and amplify paths with frontal incidence such that the Doppler spread is reduced.

In communications systems antennas with nearly omnidirectional patterns are widely used at the mobile station due to its variable position. Thus, an omnidirectional antenna is used as a reference antenna for the channel characterisation and compared to the behaviour of a directional mobile antenna.

In Figs. 3 to 6 the delay spread and Doppler spread versus snapshot time for both scenarios are presented. The light green line and dark blue line represent the results with an omnidirectional antenna and a directional antenna, respectively.

In Fig. 3 the delay spread values of the directional antenna are distinctly lower than those of the omnidirectional antenna. Except for snapshot times of approximately $0.9 \mathrm{~s}$. In this region the curve of the omnidirectional antenna experiences a deep dip. The delay spread values fall below those of the directional antenna curve. This is due to the fact that the mobile station passes at this snapshot time the base station situated at $1000 \mathrm{~m}$. For the omnidirectional antenna case the direct path is very strong at this position and experiences only a short delay. Thus, the delay spread declines for these snapshot times. However, the notch can not be recognised for the directional antenna because the antenna pattern with its pattern lobes and nulls weights the paths depending on their AoA at the mobile station. When investigating the propagation paths in the snapshots for both scenarios it can be seen that the dominant paths arrive within two main angular ranges at the mobile station (see also [12]). There are paths arriving frontally and paths that result from backward incidence. As derived in equation (1), the Doppler shift of each path depends on its AoA. The frontal incidence yields maximum positive Doppler shift, the rays from the back maximum negative Doppler shift. With a train velocity of $400 \mathrm{~km} / \mathrm{h}$ and $5.2 \mathrm{GHz}$ transmit frequency, this leads to a theoretical maximum value of the Doppler spread of $4000 \mathrm{~Hz}$.

The Doppler spread shown in Fig. 5 reduces considerably when using a directional antenna. As already seen for the delay spread, the Doppler spread values of both antennas reach the same order of magnitude when the omnidirectional antenna curve reaches a low point. This is again due to passing the second base station at $1000 \mathrm{~m}$. Figs. 4 and 6 show the delay spread and Doppler spread curves versus snapshot time for the vegetation scenario. In this case, the delay spread for both antennas ranges within the same order of magnitude. Nevertheless, the Doppler spread curves exhibit a significant improvement, i.e. lower values, for the directional antenna. Analogue to the noise barrier scenario the notches for the delay spread and Doppler spread values appear in the omnidirectional antenna curves.

As mentioned before, some propagation paths are amplified by a directional pattern, others are attenuated. The question arises if the positive effect of reducing the Doppler spread results in a decreased receive power.

In Figs. 7 and 8 the receive power of the omnidirectional and the directional antenna are plotted for the noise barrier and the vegetation scenario, respectively. The transmit power is $0 \mathrm{~dB}$. It is clearly shown that the directional pattern increases the receive power because of the antenna gain. In the first part of the simulation (up to approx. 0.6s) the increase in receive power is approx. $10 \mathrm{~dB}$ compared to the omnidirectional antenna which corresponds to the antenna gain. I.e. the main propagation paths are within the main lobe of the antenna pattern. When the mobile station is very close to the second base station the receive power is in the same order of magnitude as the omnidirectional antenna. After passing the second base station the receive power for the directional antenna rises again with a difference between the curves of approx. $5 \mathrm{~dB}$. Therefore, even if some paths are attenuated by the pattern the overall receive power 


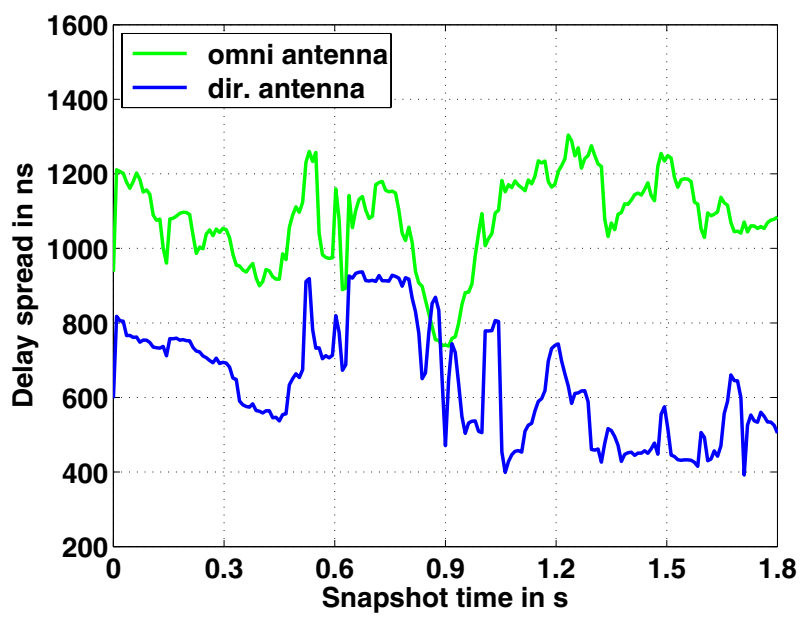

Fig. 3. Delay spread, noise barrier scenario

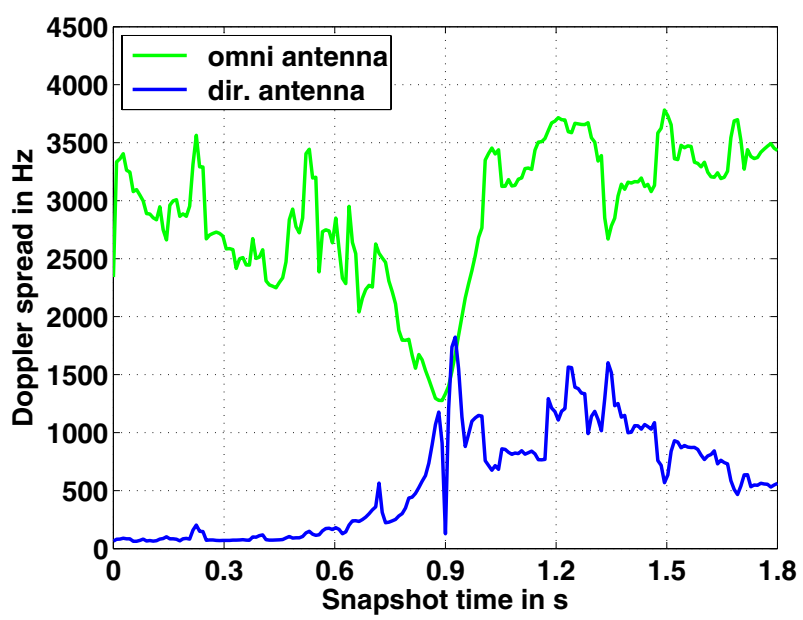

Fig. 5. Doppler spread, noise barrier scenario

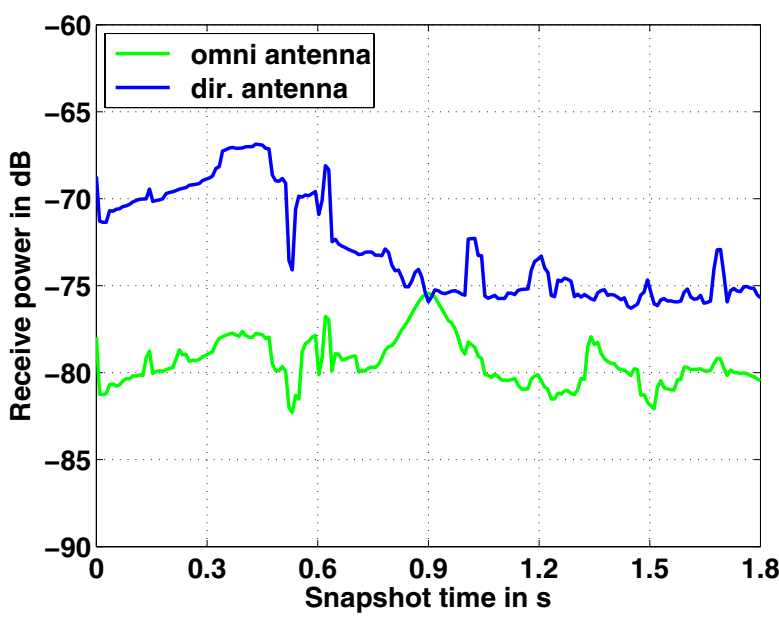

Fig. 7. Receive power normalised to transmit power, noise barrier scenario

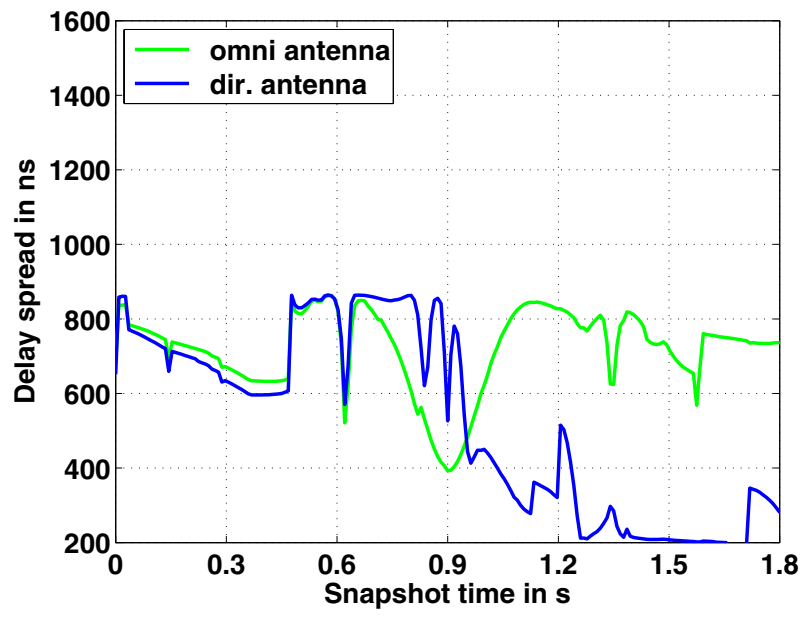

Fig. 4. Delay spread, vegetation scenario

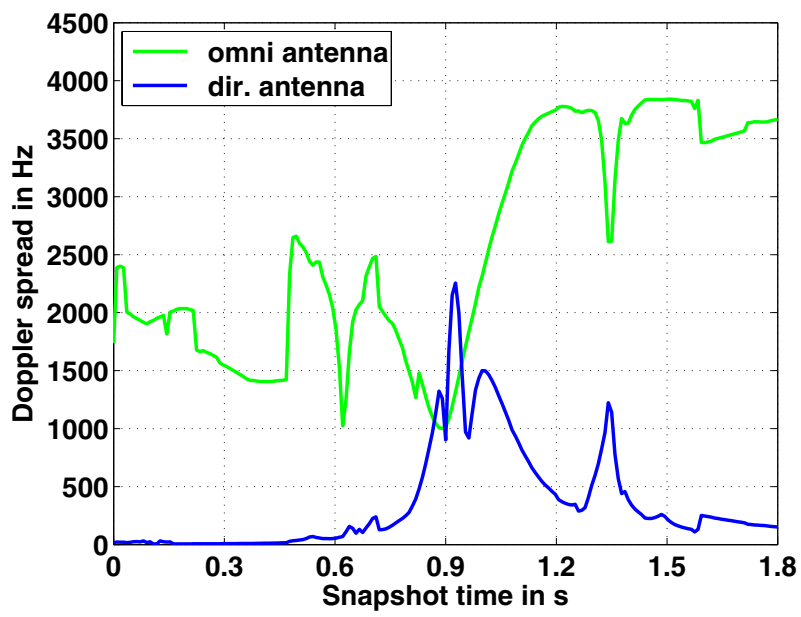

Fig. 6. Doppler spread, vegetation scenario

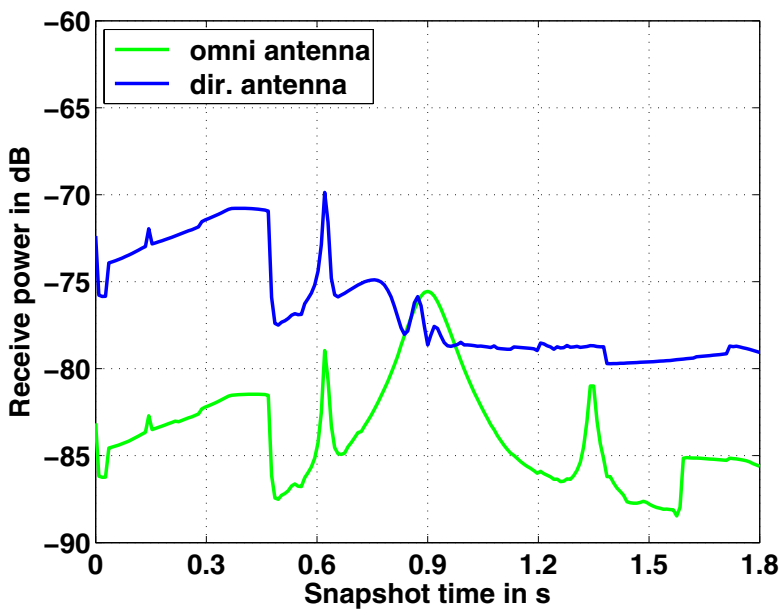

Fig. 8. Receive power normalised to transmit power, vegetation scenario 
TABLE I

200 m SIMULATION DISTANCE, THREE BASE STATIONS

\begin{tabular}{c|c|c|c|c|c|c} 
& \multicolumn{2}{|c|}{ time-average delay spread $\overline{\sigma_{\tau}}$} & \multicolumn{2}{c}{ time-average Doppler spread $\overline{\sigma_{f_{\mathrm{D}}}}$} & \multicolumn{3}{c}{ median receive power $P_{\mathrm{R}, \text { med }}$} \\
& noise barrier & vegetation & noise barrier & vegetation & noise barrier & vegetation \\
\hline $\begin{array}{c}\text { omnidirectional } \\
\text { antenna } \\
\text { directional } \\
\text { antenna }\end{array}$ & $1077.5 \mathrm{~ns}$ & $719.7 \mathrm{~ns}$ & $2889.0 \mathrm{~Hz}$ & $2574.7 \mathrm{~Hz}$ & $-79.8 \mathrm{~dB}$ & $-84.3 \mathrm{~dB}$ \\
& $641.5 \mathrm{~ns}$ & $525.3 \mathrm{~ns}$ & $570.2 \mathrm{~Hz}$ & $356.4 \mathrm{~Hz}$ & $-74.1 \mathrm{~dB}$ & $-77.8 \mathrm{~dB}$
\end{tabular}

is still higher (except a very small time span) compared to the omnidirectional antenna.

To compare the channel parameters quantitatively, Table I lists the time-average delay spread, time-average Doppler spread and median receive power for both scenarios and antennas. On the one hand, the vegetation scenario is more suitable for communications as the time-average delay spread is lower. This is due to the fact that there are less propagation paths with multiple interactions in the vegetation scenario. Also the Doppler spread is diminished for the vegetation scenario compared to the noise barrier scenario. On the other hand, the median receive power is smaller compared to the noise barrier scenario when using the same antenna. Nevertheless, the median receive power of the directional antenna for the vegetation scenario is higher than the value of the omnidirectional antenna for the noise barrier scenario. Therefore, the use of the directional antenna improves the median receive power even for the adverse scenario.

In total, a clear advantage in system performance can be derived for the directional antenna. The time-average Doppler spread reduces to a fraction of the value for the omnidirectional antenna whereas the receive power inceases. For an OFDM communications system minimal Doppler spread is aspired. If the system parameters are optimised for the channel the OFDM system allows an increased data rate when the Doppler spread decreases and the demanded bit error rate remains constant.

\section{CONCLUSION}

A channel model for high-speed train communications yields the relevant parameters for two typical scenarios. In the model a synchronised common-frequency network with three base stations and one mobile station (MISO) is simulated. This paper shows for a realistic scenario that the Doppler spread using a directional antenna is reduced and the receive power is increased compared to the omnidirectional antenna. These results imply that the channel impulse responses including the antennas can be optimised to reduce the ISI and particularly the ICI in OFDM communications systems. Therefore, assuming a fixed bit error rate the system performance can be improved considerably by smart utilisation of directional antennas at the mobile station.

\section{REFERENCES}

[1] S.B. Weinstein and P.M. Ebert, "Data transmission by frequency-division multiplexing using the discrete fourier transform," IEEE Transactions on Communications, vol. 19, no. 5, pp. 628-634, Oct. 1971.

[2] M. Russell and G.L. Stuber, "Interchannel interference analysis of OFDM in a mobile environment," IEEE $42^{\text {th }}$ Vehicular Technology Conference, vol. 2, pp. 820-824, July 1995.

[3] M. Engels, Wireless OFDM systems: How to make them work?, Boston Kluwer Academic Publishers, 2002.

[4] A. Peled and A. Ruiz, "Frequency domain data transmission using reduced computational complexity algorithms," IEEE International Conference on Acoustics, Speech, and Signal Processing, vol. 5, pp. 964-967, Apr. 1980.

[5] S. Vogeler, L. Broetje, P. Klenner, V. Kuehn, and K.D. Kammeyer, "Intercarrier interference suppression for OFDM transmission at very high velocities," 9th International OFDM Workshop (InOWo), pp. 81-85, Sept. 2004.

[6] S. Vogeler, P. Klenner, and K.D. Kammeyer, "Mulitcarrier transmission for scenarios with high Doppler influence," 10th International OFDM Workshop (InOWo), Aug. 2005.

[7] C.A. Balanis, Antenna Theory, Analysis and Design, $2^{\text {nd }}$ edition, Chapter 6 and 7, John Wiley \& Sons, Inc., 1997.

[8] V. Degli-Esposti, "A diffuse scattering model for urban propagation prediction," IEEE Transactions on Antennas and Propagation, vol. 49, no. 7, pp. 1111-1113, July 2001.

[9] S. Knörzer, J. Maurer, S. Vogeler, K.-D. Kammeyer, and W. Wiesbeck, "Channel modeling for a high-speed train OFDM communication link supporting high data rates," International Conference on ITS Telecommunications, pp. 333-336, June 2005.

[10] J. Maurer, S. Knörzer, and W. Wiesbeck, "Ray tracing in rich scattering environments for mobile-to-mobile links," International Conference on Electromagnetics in Advanced Applications, pp. 1073-1076, Sept. 2005.

[11] T. Fügen, J. Maurer, T. Kayser, and W. Wiesbeck, "Capability of 3-D ray tracing for defining parameter sets for the specification of future mobile communications systems," IEEE Transactions on Antennas and Propagation, vol. 54, no. 11, pp. 3125-3137, Nov. 2006.

[12] S. Knörzer, M.A. Baldauf, J. Maurer, and W. Wiesbeck, "OFDM for multimedia applications in high-speed trains: Channel model including different antenna types," $3^{\text {rd }}$ International Workshop on Intelligent Transportation, pp. 77-82, Mar. 2006. 